\title{
Quantum correlations in continuos-time quantum walks of two indistinguishable particles
}

\author{
Claudia Benedetti, ${ }^{1}$ Fabrizio Buscemi, ${ }^{2}$, 田 and Paolo Bordone ${ }^{3}$ \\ ${ }^{1}$ Dipartimento di Fisica, Università degli Studi di Milano, Via Celoria 16, Milano I-20133 Italy \\ ${ }^{2} A R C E S$ and Dipartimento di Elettronica, Informatica, e Sistemi, \\ Università di Bologna, Viale Risorgimento 2, I-40136 Bologna, Italy \\ ${ }^{3}$ Dipartimento di Fisica, Università di Modena e Reggio Emilia, and Centro S3, \\ CNR-Istituto di Nanoscienze, Via Campi 213/A, Modena I-41125, Italy
}

\begin{abstract}
We evaluate the degree of quantum correlation between two fermions (bosons) subject to continuous time quantum walks in a one-dimensional ring lattice with periodic boundary conditions. In our approach, no particle-particle interaction is considered. We show that the interference effects due to exchange symmetry can result into the appearance of non-classical correlations. The role played onto the appearance of quantum correlations by the quantum statistics of the particles, the boundary conditions, and the partition of the system is widely investigated. Quantum correlations also been investigated in a model mimicking the ballistic evolution of two indistinguishable particles in a $1 \mathrm{D}$ continuous space structure. Our results are consistent with recent quantum optics and electron quantum optics experiments where the showing up of two-particle non-classical correlations has been observed even in the absence of mutual interaction between the particles.

PACS numbers: 05.40.-a, 03.65.Ud, 03.67.-a
\end{abstract}

\section{INTRODUCTION}

Quantum walks (QW) describe the random walk behavior of a quantum particle [1]. Due to quantum mechanics effects, such as the coherent superposition of wavefunctions and interference, QW exhibit a qualitatively different behavior with respect to classical random walks, such as the ballistic propagation of the wavefunction instead of the diffusive behavior exhibited by the classical probability distribution [2]. Quantum effects make QW extremely promising for the implementation of more efficient and faster research algorithms than the protocols commonly adopted in the classical computation [3-5]. Due to their potential application in quantum information science, simple models of QW have been investigated and physically implemented in various physical systems ranging from quantum optics $[6-8]$ to nuclear magnetic resonance setups $[9,10]$.

Two kinds of QW are considered in the literature: discrete-time quantum coined walks and continuous-time quantum walks (CTQW). In the former, a two-level state, the so-called coin, rules the unitary discrete-time evolution of a particle moving in a lattice of sites. On the other hand, for CTQW the evolution of the particle is continuous in time and it is only determined by a Hamiltonian whose terms represent transitions among the lattice sites. CTQW have successfully been implemented in lattice waveguide systems [6 [8], where the appearance of non-classical correlations has been observed for two-photon input states [6, 8]. Indeed, due to indistinguishability of the photons, probability amplitudes of two-particle wavefunctions can interfere, thus leading

\footnotetext{
* fabrizio.buscemi@unimore.it
}

to the formation of correlations with no particle-particle interaction. Interference effects have also been successfully detected in the so-called electron quantum optics experiments, namely quantum optics experiments-like with electrons in solid state systems, where many-particle effects play a key role [11, 12]. Recent developments in nanofabrication technology have allowed exploiting the ballistic electron transport along chiral edge states at integer quantum Hall regime for the experimental realization of Mach-Zehnder and Hanbury Brown-Twiss interferometers [11, 12]. In these studies, two-particle Aharanov-Bohm oscillations, due to exchange symmetry, have been related to the degree of quantum correlation between the charge carriers, even if electron-electron interaction is not taken into account [13].

In the last decade, the notion of quantum correlations (QC) as a fundamental source for the implementation of quantum information algorithm and commonly referred as entanglement has been widely investigated in systems of identical particles [14 18]. Indeed, its quantification is certainly crucial for understanding a number of physical phenomena involving correlated indistinguishable subsystems. The main difficulties appearing in the definition of a criterion able to classify and quantify the amount of QC among indistinguishable particles are closely related to exchange simmetry which requires the symmetrization or the antisymmetrization of the quantum wavefunctions describing bosons or fermions, respectively. Different approaches have been used to estimate the degree of non-classical correlation in bipartite systems of identical particles [14-16]. The Schliemann criterion relies on the fermionic analogous of the Schmidt decomposition, namely the Slater decomposition [14]. In the approach developed by Zanardi [15], the entanglement is evaluated in terms of the QC between modes by mapping the Fock space of the modes themselves into qubit states. In the 
criterion proposed by Wiseman and Vaccaro [16, 18], the entanglement of the particles is a sort of accessible entanglement, i.e. the maximum amount of non-classical correlations which can be extracted from the system by means of local operations and then transferred into conventional quantum registers.

While the correlation between position and coin degrees of freedom of particles has been widely investigated in discrete-time quantum walks [1, 19 21], an exhaustive analysis of the building up of the QC in CTQW of identical particles is still lacking. The appearance of non-classical correlations in two-particle quantum optics CTQW setups has been discussed only qualitatively [6, 8]. The aim of this work is to provide an accurate analysis of the quantum correlation created in the CTQWs of two identical particles (both fermions and bosons) in a one-dimensional (1D) system. Specifically, we will first examine the diffusion of two fermions (bosons) in a lattice of sites with periodic boundary conditions, in order to study the development of nonclassical correlations in simple models mimicking experimental quantum optics and electron quantum optics setups. Then, we will analyze such a system in the limit of a large number of lattice sites, with a vanishing intersite distance, thus resulting into the free propagation of two identical particles along a 1D structure.

Our model does not take into account any kind of particle-particle interaction, so that the time evolution of the two-particle quantum state involving exchange symmetry is essentially ruled by single-particle Hamiltonians describing CTQW. In this way, the appearance of QC is only related to two-fermion (-boson) amplitudes interference due to the quantum statistics of the particles involved in the process. In this work, in order to estimate the degree of non-classical correlation between the positions of the particles, we adopt the criterion proposed by Wiseman and Vaccaro [16, 18]. In fact, such a criterion, unlike the approach developed by Schliemann [14], behaves correctly under one-site (local) and two-site (non local) transformations. Furthermore, its use does not lead to the violation of the local number of particle superselection rule, as may happen in the case of the procedure proposed by Zanardi [15].

The paper is organized as follows. In Sec. III we describe the theoretical approach used to quantify the QC between two indistinguishable particles. In Sec. III, we study the CTQW of two fermions (bosons) in a lattice of sites with periodic boundary conditions and then estimate the time evolution of the amount of $\mathrm{QC}$ of the system. In Sec. IV], we numerically evaluate the degree of non classical correlation between two identical particles free propagating in a 1D structure. Finally, conclusions and discussions are given in Sec. V.

\section{EVALUATOR OF THE DEGREE OF QUANTUM CORRELATIONS IN BIPARTITE SYSTEMS OF IDENTICAL PARTICLES}

Here, we briefly illustrate the theorethical criterion adopted to evaluate the degree of quantum correlation in two-fermion and -boson systems. It is based on the notion of entanglement of particles proposed by Wiseman and Vaccaro [16, 18].

By using the mode-occupation representation, an arbitrary pure two-fermion (boson) state in a $M$-mode system can be expressed as

$$
|\Psi\rangle=\sum_{\{n\}} c_{\{n\}}|\{n\}\rangle,
$$

where the integers $n_{i}$ of the set $\{n\}=n_{1}, \ldots, n_{i}, \ldots, n_{M}$ satisfy the relation $n_{1}+\ldots+n_{i}+\ldots+n_{M}=2$. Here, the ket $|\{n\}\rangle$ indicates the state vector in the Fock space with $n_{i}$ 's particles in the $i$-th mode, and the $c_{\{n\}}$ 's are the coefficients of the linear superposition. While for bosons $n_{i}$ 's range from 0 to 2 , for fermions the occupations numbers are restricted to be 0 or 1 due to the Pauli exclusion principle.

As argued by some authors [15], a formal equivalence between the space of the occupation-number states and the tensor product space of the modes can be established. In this way, the occupation number of each mode constitutes a distinct state of the mode itself. In the Zanardi approach [15], the amount of non-classical correlations between the occupation numbers of the modes controlled by two parties of the system, namely Alice and Bob, represents the so-called entanglement of modes. The latter does not always constitute a valid measure of the true degree of quantum correlation between Alice and Bob [18]. As a matter of fact, not only the entanglement of modes can give values different from zero even when applied to suitable single-particle states [22], but also it does not take into account the local-particle number superselection rule (LPNSR) [16]. Indeed to fully exploit the QC between modes, Alice and Bob, at least in principle, must be able to arbitrarily measure and manipulate their local systems. Unless each party of the systems possesses a definite number of particles, this will lead to a violation of the LPNSR.

The Wiseman and Vaccaro criterion satisfies the LPNSR. In such an approach, in addition to the two identical particles shared by Alice and Bob, their quantum state $|\Psi\rangle$ involve a standard quantum register, namely a set of distinguishable qubits. The entanglement of the particles $E_{P}$ is defined as the maximum amount of QC that Alice and Bob can produce between their standard quantum registers by means of local operations. As a consequence of the LPNSR, $E_{P}$, in place of the QC between the modes that Alice and Bob have access to, is given by

$$
E_{P}(|\Psi\rangle)=\sum_{n=0}^{2} P_{n} E_{M}\left(\left|\Psi^{(n)}\right\rangle\right),
$$


where $\left|\Psi^{(n)}\right\rangle$ is the projection of the quantum state $|\Psi\rangle$, describing the global system, onto the Fock subspace where Alice controls $n$ particles and Bob the remaining $(2-n)$ ones. $P_{n}=\left\langle\Psi^{(n)} \mid \Psi^{(n)}\right\rangle$ is the probability for Alice (Bob) of finding $n(2-n)$ particles as a consequence of a measure of the local number of particles, while $E_{M}$ is the degree of quantum correlation between the two sets of modes, each controlled by a party of the system. In other terms, $E_{P}$ is the weighted sum of the entanglement of modes when the local particle number is measured. It is worth noting that $E_{P}$ depends upon the partition of the system, that is upon which modes Alice and Bob control. From this point of view, different partitions of the system can lead to different values of $E_{P}$.

For two-particle pure states, the expression given in Eq. (2) takes a simple form. Indeed, only some quantum states $\left|\Psi^{(n)}\right\rangle$, belonging to the Fock subspace with a fixed local number of particles, give a non vanishing contribution to entanglement. Specifically, let us consider the case of the set of states $\left|\Psi^{(0)}\right\rangle$ where the local number of particles possessed by Alice is zero. Any state of such a set can be written, in the mode occupation number, as $\left|0_{n_{A}}\right\rangle \otimes\left|2_{n_{B}}\right\rangle$ and it is separable, that is it can be factorized in a term describing Alice with no particle and in a term describing Bob with two particles. This implies that the contribution of $\left|\Psi^{(0)}\right\rangle$ to $E_{P}$ is zero. Analogously, the amount of quantum correlations stemming from set of states $\left|\Psi^{(2)}\right\rangle$ is also zero. This implies that the bipartite entanglement is non-vanishing only when both Alice and Bob have one particle, that is only for the quantum states belonging to the set $\left|\Psi^{(1)}\right\rangle$. As shown in Ref. [16], $E_{P}$ becomes

$$
E_{P}=P_{1} \epsilon\left[\rho_{A}^{(1)}\right]
$$

where $\epsilon$ indicates an arbitrary quantum binary entropy and

$$
\left[\rho_{A}^{(1)}\right]_{k k^{\prime}}=\frac{\left\langle\Psi^{(1)}\left|a_{k}^{\dagger} a_{k^{\prime}}\right| \Psi^{(1)}\right\rangle}{\sum_{l}\left\langle\Psi^{(1)}\left|a_{l}^{\dagger} a_{l}\right| \Psi^{(1)}\right\rangle}
$$

is the single-particle matrix describing the subsystem controlled by Alice. The latter is obtained from $\left|\Psi^{(1)}\right\rangle$ by means of the creation (annihilation) operators $a_{k}^{\dagger}\left(a_{k}\right)$ acting on Alice's modes only. The form of $E_{P}$ given in Eq. (3) holds for either bosons or fermions.

\section{TWO-PARTICLE CONTINUOUS-TIME QUANTUM WALKS ON 1D RING LATTICES}

In this section, a model of two-fermion and two-boson CTQW in 1D graphs is solved by means of analytical and numerical techniques for a small and large number of nodes, respectively. The time evolution of the wavefunction describing the system is then used to estimate the degree of quantum correlation according to the criterion given in Eq. (3).

Here we examine CTQW on a 1D ring lattices of $N$ sites (with $N$ even) with periodic boundary condi- tions. In agreement with previous single-particle investigations 23], the topology of the graphs here considered is simple in the sense that each node is connected to its two first neighbours. Even if more complex networks, among which two-dimensional lattices [24] or graphs with larger connectivity [25], could be examined, our model is good enough to describe experimental implementations of CTQW, such as two-photon transport in an array of waveguide lattices where non-classical correlations appear. Therefore, though simple, it represents a valid tool to analyze the amount of $\mathrm{QC}$ appearing in two-particle CTQW.

The two-particle Hamiltonian describing the dynamical evolution of the system is given by

$$
\mathcal{H}=\mathcal{H}_{\alpha}^{0}+\mathcal{H}_{\beta}^{0},
$$

where $\mathcal{H}_{\alpha(\beta)}^{0}$ is the single-particle Hamiltonian acting on the particle $\alpha(\beta)$ :

$$
\mathcal{H}_{\alpha(\beta)}^{0}|j\rangle_{\alpha(\beta)}=\gamma\left(2|j\rangle_{\alpha(\beta)}-|j-1\rangle_{\alpha(\beta)}-|j+1\rangle_{\alpha(\beta)}\right) .
$$

$|j\rangle_{\alpha(\beta)}$ indicates the quantum state describing the particle $\alpha(\beta)$ localized in the $j$ node and forming a complete, orthonormalized basis set, which span the whole accessible Hilbert space. $\gamma$ denotes the intersite transmission rate. Due to the periodic boundary conditions, here we assume that the node $N+1$ coincides with node 1. Eq. (6) is the discrete version of the Laplacian, and, in turns, the discrete version of the Hamiltonian describing the free propagation of a particle in a lattice.

Given the form of the two-particle Hamiltonian in Eq. (5), single-particle dynamics allows one to estimate the time evolution of the two-fermion and -boson system in the basis states given by

$$
|j k\rangle_{f}=\frac{1}{\sqrt{2}}\left(|j k\rangle_{\alpha \beta}-|k j\rangle_{\alpha \beta}\right),
$$

and

$$
|j k\rangle_{b}= \begin{cases}\frac{1}{\sqrt{2}}\left(|j k\rangle_{\alpha \beta}+|k j\rangle_{\alpha \beta}\right): & j \neq k, \\ |j j\rangle_{\alpha \beta}: & j=k,\end{cases}
$$

respectively. Specifically in order to solve the model, which can be interpreted as the free propagation of two identical particles in a periodic system, we adopt the Bloch function approach, in analogy with the method commonly used in solid-state physics [23]. The singleparticle Bloch states

$$
\left|\phi_{n}\right\rangle=\frac{1}{\sqrt{N}} \sum_{j=1}^{N} \exp \left(-i \frac{2 \pi n}{N} j\right)|j\rangle,
$$

are eigenstates of the Hamiltonian of the Eq. (6) with eigenvalues $E_{n}=2 \gamma\left(1-\cos \frac{2 \pi n}{N}\right)$, and can be used to evaluate the coefficients describing the transition amplitude $\lambda_{k, j}(t)=\left\langle k\left|e^{-i H_{0} t}\right| j\right\rangle$ from the state $|j\rangle$ at $t=0$ to state $|k\rangle$ at time $t$. Indeed the latter can be expressed as: 


$$
\begin{aligned}
\lambda_{k, j}(t) & =\sum_{n=1}^{N} \sum_{m=1}^{N}\left\langle k \mid \phi_{n}\right\rangle\left\langle\phi_{n}\left|e^{-i H_{0} t}\right| \phi_{m}\right\rangle\left\langle\phi_{m} \mid j\right\rangle \\
& =\frac{\exp (-2 i \gamma t)}{N} \sum_{n=1}^{N} \exp \left(2 i \gamma t \cos \left(\frac{2 n \pi}{N}\right)\right) \exp \left(-i \frac{2 \pi n}{N}(k-j)\right),
\end{aligned}
$$

where is set $\hbar=1$.

By using the two-particle basis states written in Eqs. (8) and (7), we can evaluate, in terms of the above single-particle coefficients, the two-boson (-fermion) transition amplitude $\mu_{k s, j r}^{f(b)}(t)$, from the state $|j r\rangle_{f(b)}$ at $t=0$ to state $|k s\rangle_{f(b)}$ at time $t$. It reads:

$$
\mu_{k s, j r}^{f}(t)=\lambda_{k, j}(t) \lambda_{s, r}(t)-\lambda_{k, r}(t) \lambda_{s, j}(t)
$$

and

$$
\mu_{k s, j r}^{b}(t)=\left\{\begin{array}{ll}
\lambda_{k, j}(t) \lambda_{s, r}(t)+\lambda_{k, r}(t) \lambda_{s, j}(t) & k \neq s \text { and } j \neq r \\
\sqrt{2} \lambda_{k, j}(t) \lambda_{s, r}(t) & k=s \text { xor } j=r \\
\lambda_{k, j}(t) \lambda_{s, r}(t) & k=s \text { and } j=r
\end{array} .\right.
$$

Once the time evolution of the two-particle quantum state $|j r\rangle_{f(b)}$ describing two fermions (bosons) initially localized in the node $j$ and $r$ is known, the dynamics of the built up of the amount of quantum correlation can be quantified by using Eq. (3). To this aim, different partitions of the system can be considered. Note that here the modes of the system correspond to the sites of the lattice. In the simplest case, Alice controls the first half of the lattice and Bob the second one, namely $A=\left\{1, \ldots, \frac{N}{2}\right\}$ and $B=\left\{\frac{N}{2}+1, \ldots, N\right\}$. Thus, the single-particle density matrix of the Alice's subsystem given in Eq. (41) takes, for the initial state $|j r\rangle_{b(f)}$, the form

$$
\left[\rho(t)_{A}^{(1)}\right]_{k k^{\prime}}=\frac{\sum_{s=\frac{N}{2}+1}^{N} \mu_{k s, j r}^{*}(t) \mu_{k^{\prime} s, j r}(t)}{\sum_{k=1}^{\frac{N}{2}} \sum_{s=\frac{N}{2}+1}^{N}\left|\mu_{k s, j r}(t)\right|^{2}} .
$$

Its quantum entropy can be quantified by means of the von Neumann entropy

$$
\epsilon_{v N}(t)=-\operatorname{Tr}\left\{\left[\rho(t)_{A}^{(1)}\right] \ln \left[\rho(t)_{A}^{(1)}\right]\right\},
$$

which represents an appropriate evaluator of the degree of the correlation in standard bipartite systems, and this can be used in Eq. (3) in order to estimate of the entanglement of particles. For sake of completeness, in the following, other possible partitions of the system will be examined, where Alice and Bob can access to non-adjacent modes. The evaluation of $E_{P}$ in such cases requires to calculate again both the single-particle density matrix of the subsystems and the probability of finding one particle in each subsystem, being the latter closely dependent upon the specific partition.

\section{A. Small number of nodes}

Here we study, by means of analytical techniques, the quantum correlation dynamics for the case of an 1D ring lattice composed of a small number of sites. Specifically, we take $N=4$. Various initial configurations of the bosonic (fermionic) system, that is the states describing at time $t=0$ the two identical particles, should be examined. However, due to the periodic boundary conditions of the lattice under investigation, the number of initial setups of the system resulting into different time evolutions of the QC turns out to be smaller than the dimension of the Fock space of the two-fermion (-boson) system.

First, let us analyze the case of particles initially localized in two different nodes for the partition $A=\{1,2\}$ and $B=\{3,4\}$. Three configurations are possible: both particles occupy the Alice (Bob) modes, that is the quantum state $|12\rangle_{f(b)}\left(|34\rangle_{f(b)}\right)$; one particle is in an Alice site and the other one is localized in the adjacent site controlled by Bob, the state $|23\rangle_{f(b)}$ or $|14\rangle_{f(b)}$, or in the nonadjacent Bob mode, the state $|13\rangle_{f(b)}$ or $|24\rangle_{f(b)}$. Thus, to analyze the role of the initial configuration of the system into the building up of non-classical correlations, we just need to evaluate the time evolution of the quantum states $|12\rangle_{f(b)},|23\rangle_{f(b)}$, and $|13\rangle_{f(b)}$. As shown in the previous section, the latter can be calculated from the single-particle amplitude transitions given in Eq. (10). In agreement with the results of Ref. [23], these, after a straightforward calculation for the case of $N=4$, can be expressed as:

$$
\begin{aligned}
\lambda_{k, k}(t) & =\exp (-2 i \gamma t) \cos ^{2} \gamma t \\
\lambda_{k \pm 1, k}(t)=\lambda_{k, k \pm 1}(t) & =\exp (-2 i \gamma t) i \sin \gamma t \cos \gamma t \\
\lambda_{k \pm 2, k}(t)=\lambda_{k, k \pm 2}(t) & =-\exp (-2 i \gamma t) \sin ^{2} \gamma t
\end{aligned}
$$


By inserting the above coefficients in the Eqs. (12) and (11), and then by using the expressions (13) and (14), we can quantify the building up of $\mathrm{QC}$ for the initial quantum states of interest. We find that

$$
\begin{aligned}
E_{P}\left(\left|\psi^{(12)}\right\rangle_{f(b)}\right)_{A=\{1,2\}}= & \frac{1}{2} \sin ^{2}(2 \gamma t) \ln 2 \\
E_{P}\left(\left|\psi^{(13)}\right\rangle_{f(b)}\right)_{A=\{1,2\}}= & -\frac{[\cos (2 \gamma t)-1]^{2}}{4} \ln \left[\frac{[\cos (2 \gamma t)-1]^{2}}{2\left[1+\cos ^{2}(2 \gamma t)\right.}\right] \\
& -\frac{[\cos (2 \gamma t)+1]^{2}}{4} \ln \left[\frac{[\cos (2 \gamma t)+1]^{2}}{2\left[1+\cos ^{2}(2 \gamma t)\right]}\right] \\
E_{P}\left(\left|\psi^{(23)}\right\rangle_{f(b)}\right)_{A=\{1,2\}}= & 0 .
\end{aligned}
$$

In Figure 1, we report the time evolution of $E_{P}$, namely the amount the QC, for a two-fermion (-boson) system prepared in different initial conditions. For the sake of simplicity, in this section we call time the adimensional parameter $\gamma t$. In agreement with theoretical predictions, at $t=0, E_{P}$ is null, thus indicating the absence of QC between the two particles localized in different nodes. After initial time, $E_{P}$ remains zero for the input state $\left|\psi^{(23)}\right\rangle_{f(b)}$, while it exhibits oscillations, between 0 and $\ln 2 / 2$, with a period of $\pi / 2$ for the other two input states $\left|\psi^{(12)}\right\rangle_{f(b)}$ and $\left|\psi^{(13)}\right\rangle_{f(b)}$. While in the former initial configuration the dynamics of the two-particle wavefunction does not result into building up of non-classical correlations, in the latter cases the interference effects are able to produce QC, whose periodic behavior is strictly related to the evolution of the system in a ring lattice with a small number of nodes with periodic boundary conditions. Here, the highest value of $E_{P}$ is $\ln 2 / 2$, that is the half of the maximum degree of quantum correlation achievable between two particles, each one in a two-mode subsystem. It is worth noting that, for the partition con-

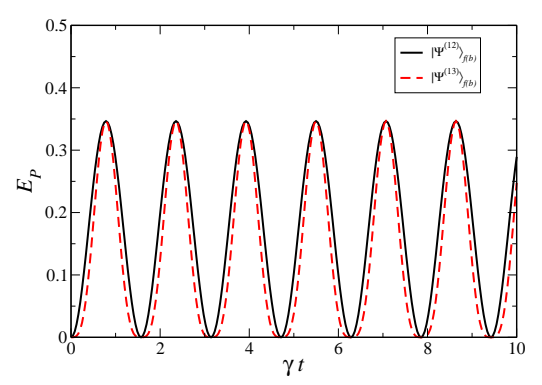

FIG. 1. (Color online) Time evolution of $E_{P}$ for three input states, namely $|12\rangle_{f(b)}$ (solid line), $|13\rangle_{f(b)}$ (dashed line), with Alice controlling sites $\{1,2\}$ and Bob $\{3,4\}$. For sake of clarity, the entanglement dynamics of the initial state $|23\rangle_{f(b)}$ has not been reported being always null.

sidered, $E_{P}$ turns out not to depend upon the quantum statistics of the particles involved in the process. Such a result is somehow unexpected due to the different number of states accessible to the two systems during the time evolution. Indeed, unlike fermions, bosons can occupy simultaneously the same node, that is the transition probability to the states $|k k\rangle_{b}$ 's does not vanish as time goes by. Even if the time evolution of the bosonic wavefunction differs from the fermionic one, for the specific partition here considered the two-particle interference effects lead to the same single-particle features, as, in particular, the reduced density matrices of the subsystems. As a consequence, also the amount of $\mathrm{QC}$ is the same.

The results above reported are closely related to how the nodes of the ring lattice are assigned to Alice and Bob. If we move from the partition $A=\{1,2\}$ and $B=$ $\{3,4\}$ to the one $A=\{1,3\}$ and $B=\{2,4\}$, not only the QC turn out to depend upon the quantum statistics of the particles, but the highest degree of correlation can also be reached. Let us examine the time evolution of two bosons, or two fermions, initially occupying the nodes 1 and 3. After a straightforward calculation, we obtain 


$$
E_{P}\left(\left|\psi^{(13)}\right\rangle_{f}\right)_{A=\{1,3\}}=\ln 2 \sin ^{2}(2 \gamma t)
$$

While in the two-boson system no correlation is created, in the two-fermion one $E_{P}$ exhibits periodic oscillations and reaches the maximum value, namely $\ln 2$, for $\gamma t=(2 k+1) /(4 \pi)$ with $k \in \mathbb{N}$.

As stated above, Bose-Einstein statistics allows for the localization of two bosons on the same site. When the latter is taken as initial configuration of the two-particle system, the amount of non-classical correlations built up does not change with time and remains equal to zero for any partition examined. This means that time-evolved states of the kind $|k k\rangle_{b}$ can always be factorized in terms of an one-particle state in the Alice modes and one in the Bob modes. Thus, an initial bosonic bunching prevents the appearance of QC in CTQW.

\section{B. Large number of nodes}

For a lattice with $N$ sites, the dimensions of the Hilbert space for the two-boson and two-fermion systems are $N(N+1) / 2$ and $N(N-1) / 2$, respectively. This makes the analytical techniques inefficient for large $N$ both to solve the two-particle dynamics and to evaluate the degree of quantum correlation. For this reason, we implemented a numerical approach that, once known the time evolution of the state, allows one first to diagonalize the single-particle reduced density matrix given in Eq. (13), and then to estimate, at any time, $E_{P}$. Such an approach is used here to quantify the amount of quantum correlations stemming from two-particle CTQW in an 1D ring lattice with $N=50,70,100$, when Alice accesses the first half of the lattice and Bob the second half (namely, the partition $A=\{1 \ldots, N / 2\}, B=\{N / 2+1, \ldots, N\})$.
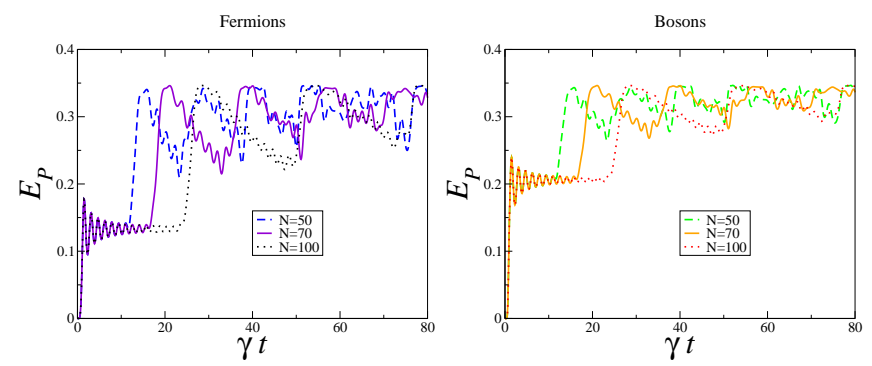

FIG. 2. (Color online) Time evolution of $E_{P}$ for the CTWQ of two fermions and two bosons, in the left and right panel, respectively, on a lattice with a number of sites $N=50$ (dashed line), $N=70$ (solid line), and $N=100$ (dotted line). Both systems are initially prepared in the state $\left|\frac{N}{2} \frac{N}{2}+1\right\rangle_{f(b)}$.

Fig. 2 displays the time evolution of $E_{P}$ for two fermions (bosons) initially localized in two adjacent sites belonging to different subsystems, corresponding to the input state $|N / 2, N / 2+1\rangle_{f(b)}$. As expected, at $t=0$ the and

$$
E_{P}\left(\left|\psi^{(13)}\right\rangle_{b}\right)_{A=\{1,3\}}=0
$$

amount of $\mathrm{QC}$ is zero. As time goes by, $E_{P}$ increases and saturates, apart from small oscillations, around a specific value for a time interval $\gamma \tau$ which is found to be linearly dependent upon the number $N$ of lattice nodes. These oscillations are closely related to the two-particle interference effects stemming from the spreading of the spatial wavepackets in a discrete lattice. In the limit of large values of $N$, the fluctuations disappear leading to a smoother behavior, as it will be shown in the next section. After a time $\gamma \tau, E_{P}$ shows first a rapid increase and then again large fluctuations around a new saturation value.

In order to get a better insight into the appearance of $\mathrm{QC}$, we focus on the time-evolution of the two-fermion (-boson) correlation function $\Gamma_{k j}^{f(b)}(t)[6]$, namely the square modulus of the coefficient $\mu_{k j, \frac{N}{2} \frac{N}{2}+1}^{f(b)}(t)$. To this aim, we report in Fig. 3 the values of $\Gamma_{k j}^{f(b)}(t)$, at four different times, for the case of a ring lattice with 70 nodes. At initial time, the two fermions (bosons) are localized in two adjacent sites (taken in the middle of the lattice) in a separable state. After a relatively short time, their wavepackets overlap and interfere thus leading to the first rise of $E_{P}$. Then the fermions (bosons) freely propagate along the lattices exhibiting spatial antibunching (bunching) 26], as shown by the two peaks positioned along the antidiagonal (diagonal) of the correlation matrix (see panels (b) and (c) of the Fig. 3). During this ballistic evolution, the amount of QC exhibits small fluctuations around a given value in the interval $\gamma \tau$. The latter can now be viewed as a characteristic parameter of the dynamics of the system. Indeed, it corresponds to the travelling time of the two particles along half a lattice before the transmission thorugh periodic boundary conditions occurs. As expected, $\gamma \tau$ is independent upon the quantum statistics of the particles. At longer times, the two-particle wavefunction is transmitted by the periodic boundary conditions, and their components interfere again creating additional QC. As reported in the panels (d) of Fig. 3), at $\gamma t=40, \Gamma_{k j}^{f(b)}$ reveals a checkered pattern where both fermions and bosons are scattered along the whole lattice and $E_{P}$ oscillates in time.

The time evolution of $E_{P}$ deserves further comments. The results reported here differ significantly from the ones found for the case of a lattice with $N=4$. First, $\mathrm{QC}$ depend now upon the quantum statistics of the the particles considered, though the qualitative behavior of $E_{P}$ of the two-fermion and -boson systems is the same. Specifically, we find that the degree of correlation between two bosons is always equal or greater than the one of the fermionic system. Such a behavior is at a first analysis counterintuitive. Unlike the two-fermion dynamics, the bosonic bunching lowers the probability $P_{1}$ of find- 
ing each of the two particles in a different subsystem (see Fig. 4). As a consequence, according to Eq. (3), the amount of QC between two bosons should be lower than the one created in the fermionic system. However, also the degree of non-classical correlation of the two-particle state describing one particle in the Alice modes and the other one in the Bob modes, and quantified by the von Neumann entropy, has to be taken into account. In this case, the latter is larger for the two-boson CTQW and the product $P_{1} \epsilon_{v N}$, namely $E_{P}$, turns out to be larger than the one corresponding to the two-fermion system.

Furthermore, we find that the behavior in time of $E_{P}$ is not periodic. Indeed, as expected, for lattices with a large number of sites, the transmission through the boundary conditions does not affect very much the time evolution of the two-particle wavefunction. As a consequence the interference among the different components of the wavefunction leads to a non-periodic behavior of the correlations Thus, the effect of the lattice periodicity on two-particle dynamics and the degree of quantum correlation is lower for lattices with higher values of $N$ and should vanish in the limit of a very large $N$.

\section{TWO-PARTICLE FREE PROPAGATION IN 1D SPACE-CONTINUOUS STRUCTURES}

In this section, we numerically study the building up of non-classical correlations in the free-propagation of two identical particles in a $1 \mathrm{D}$ structure.

The physical model here investigated consists of two non-interacting massive fermions (bosons) travelling in a space-continuous structure. The latter can be viewed as an infinite lattice with vanishing intersite distance without the interference effect stemming from the transmission through the periodic boundary conditions. In other terms, we are considering the continuous-space limit of the model examined in the previous section. If the free propagation of massive particles obeying the BoseEinstein statistics constitutes a helpful theoretical tool to investigate the appearance of $\mathrm{QC}$ due to the bunching in a space-continuous structure, on the other hand the fermionic system can also describe phenomena of physical interest, like the electron diffusion in solid state systems.

The dynamics of the global system can be described by the two-particle Hamiltonian:

$$
\mathcal{H}\left(x_{1}, x_{2}\right)=-\frac{\hbar^{2}}{2 m}\left(\frac{\partial^{2}}{\partial x_{1}^{2}}+\frac{\partial^{2}}{\partial x_{2}^{2}}\right)
$$

with $m$ indicating the particle mass. Eq. (18), that is the sum of two Laplacians, is the continuous version of the Hamiltonian written in Eq. (5) for the CTQW of two particles. Both of them are represented, at the initial time $t_{0}$, by a minimum-uncertainty wavepacket, that in the space representation, reads:

$$
\phi_{ \pm x_{0}, k}\left(x, t_{0}\right)=\left(\frac{1}{2 \pi \sigma^{2}}\right)^{1 / 4} \exp \left(i k x-\frac{\left(x \mp x_{0}\right)^{2}}{4 \sigma^{2}}\right)
$$

where $\pm x_{0}$ is the mean position of the space wavepacket with variance $\sigma$, and $k=\frac{\sqrt{2 m E_{k}}}{\hbar}$ with $E_{k}$ kinetic energy of the particle. Thus the two-fermion (-boson) initial wavefunction $\Phi_{f(b)}\left(x_{1}, x_{2}\right)$ can be written as:

$$
\Phi_{f(b)}\left(x_{1}, x_{2}, t_{0}\right)=\phi_{\bar{x}_{0}, k_{1}}\left(x_{1}, t_{0}\right) \phi_{-\bar{x}_{0}, k_{2}}\left(x_{2}, t_{0}\right)-(+) \phi_{\bar{x}_{0}, k_{1}}\left(x_{2}, t_{0}\right) \phi_{-\bar{x}_{0}, k_{2}}\left(x_{1}, t_{0}\right),
$$

with the normalization condition $\int_{-\infty}^{+\infty} d x_{1} \int_{x_{1}}^{+\infty} d x_{2}\left|\Phi_{b(f)}\left(x_{1}, x_{2}, t_{0}\right)\right|^{2}=1$. The variance $\sigma$ of the two single-particle Gaussian wavepackets and the distance $\left|2 \bar{x}_{0}\right|$ between their centers are such that, at the initial time, their spatial overlap is practically null.

Here we estimate the amount of QC between the spatial degrees of freedom of the two particles. Specifically, we assume that the space domain $[-\infty, 0]$ is controlled by Alice, while Bob controls to the spatial modes in the interval $[0,+\infty]$. To quantify $E_{P}$ we adopt a numerical procedure. In fact, even if the dynamics of the system could be evaluated analytically, nevertheless the estimation of the time evolution of $E_{P}$ as given in Eq. (3) would require involved calculations. On the contrary, the implementation of a numerical approach, exploiting the Crank-Nicholson finite difference scheme to solve the two-particle time-dependent Schrödinger equation, permits to evaluate efficiently, at fixed time steps, the built up of the non-classical correlations. Here $E_{P}$ is evaluated by means of the linear entropy $\epsilon_{L}$, in place of the von Neumman entropy, in order to make the numerical procedure less demanding, as shown in Ref. 27. Given the relation $\epsilon_{L}=1-\operatorname{Tr}\left(\rho_{A}^{2}\right), E_{P}$ reads

$$
E_{P}=P_{1}\left(1-\operatorname{Tr}\left(\rho_{A}^{2}\right)\right)=P_{1}\left(1-\int_{-\infty}^{0} d x \rho_{A}^{2}(x, x)\right),
$$

where the space-continuous nature of the model is explicitly taken into account. In the above expression, the probability $P_{1}$ of finding one particle in the Alice side and the other in the Bob side, is given by $\int_{-\infty}^{0} d x_{1} \int_{0}^{+\infty} d x_{2}\left|\Phi_{b(f)}\left(x_{1}, x_{2}, t\right)\right|^{2}$, while the single-particle density matrix describing the subsystem controlled by Alice is $\rho_{A}\left(x, x^{\prime}\right)=$ $\left(1 / P_{1}\right) \int_{0}^{+\infty} d x^{\prime \prime} \Phi_{b(f)}\left(x, x^{\prime \prime}, t\right) \Phi_{b(f)}^{*}\left(x^{\prime}, x^{\prime \prime}, t\right)$ with $x, x^{\prime} \in$ $[-\infty, 0]$.

We examine the model for two different initial condi- 
tions: particles with the same velocity, that is $k_{1}=k_{2}$, and particles with opposite velocities, that is $k_{1}=-k_{2}$. The latter case mimics a collision event between two non interacting identical particles.

The former case can be thought as the spacecontinuous analogous of the CTQW on a site lattice with two particles initially localized in specific sites. At the initial time, both Alice and Bob have a particle: the centers of the two single-particle wavefunctions are located in different spatial subdomains and the space overlap between the wavefunctions is zero. This makes the degree of non-classical correlation vanishing, as shown in left panel of Fig. 5. As time increases, single-particle wavepackets spread out making the spatial overlap between them not negligible anymore. Now the probability amplitudes of the two-fermion (-boson) wavefunction $\Phi_{b(f)}\left(x_{1}, x_{2}\right)$ can interfere and quantum correlation builds up. The latter increases with time ad finally reaches a stationary value depending upon the quantum statistics of the particles involved: it is higher for bosons. Apart from the absence of oscillations which can be related to the spacecontinuous structure, the time evolution of $E_{P}$ of the fermionic (bosonic) system is, at least at short times, in qualitative agreement with the dynamics of the quantum correlations appearing in the model investigated in the previous section. At longer times, due to the different boundary conditions of the two systems, a discrepancy between the two entanglement-time behavior is observed. While in the ring lattice the two-particle wavefunction is transmitted through periodic boundary conditions and this results in a further increase of the amount of quantum correlations, the space-continuous structure is supposed to be infinite and the wavepackets can only spread with no additional interference effect due to transmitted components.

For the case of two fermions (bosons) running in opposite directions, $\mathrm{QC}$ exhibit a peculiar behavior (see the right panel of Fig. (5). $E_{P}$ increases while the two fermions (bosons) are approaching each other. Specifically, when the centers of the two wave packets reach the minimum distance, two-particle interference gives the maximum amount of quantum correlations. Finally, $E_{P}$ drops again to zero once the particles get far apart and the corresponding Gaussian wave packets exhibit a negligible spatial overlap. This behavior is in agreement with previous analyses, adopting other entanglement criteria, of the dynamics of the QC stemming from carrier-carrier scattering events in semiconductor nanostructures [28, 29].

\section{CONCLUSIONS}

Recent experiments addressed the appearance of nonclassical correlations in CTWQ of photon pairs in coupled waveguide lattices [6, 8]. Such correlations have been related to non trivial interference effects due to the quantum statistics of the particles. In this perspective, an accurate analysis of the quantum correlation created in few-particle CTQW models is undoubtedly of great interest given their experimental feasibility and potential application in quantum communication and quantum computation theory [ $[6,8]$.

Here, we investigated the appearance of QC in CTWQ of two non-interacting bosons (fermions) on an 1D ring lattice with periodic boundary conditions. Specifically, in our model the topology of the graphs examined is very simple, that is each node is connected to its first neighbours and this permits to express the Hamiltonian, ruling the two-particle dynamics, in terms of the so-called discrete single-particle Laplacian. Indeed, our CTQW well describes the free propagation of two non-interacting identical particles in a periodic system. Given the key role played by the exchange symmetry into the emergence of non-classical correlations, their quantitative evaluation in our physical system required the use of a suitable criterion which takes into account the indistinguishability of the particles. To this purpose we adopted the Wiseman and Vaccaro approach [16] which allowed estimating the degree of quantum correlation between two parties of the system (each possessing one particle and accessing to a given set of nodes) with no violation of the particle local number superselection rules, as occurring in the preparation, manipulation and measurement of the experimental implementations of CTQW.

In agreement with the theoretical predictions [ $[6,8]$, results indicate that the building up of $\mathrm{QC}$ in our system is due to the two-particle interference effects between the propagating wavepackets. Indeed, the time evolution of the quantum system affects the degree of correlation. Specifically, we find that, for CTQW on discrete ring lattices with a small number of nodes, the transition amplitudes depend sinusoidally on time due to the transmission of the wavepackets through periodic boundaries conditions. As a consequence, also QC exhibit a periodic-time behavior. They are also affected by the quantum statistics of the particles involved in the process and by the partition of the system. Specifically, the production of the maximum degree of non-classical correlation between subsystem accessing non adjacent nodes occurs, cyclically, only for two-fermion systems initially prepared in a suitable input state. Such a result seems consistent with experiments in electron Hanbury BrownTwiss interferometers showing the emergence of QC between two identical charge carriers at couples of non adjacent drains [12, 13]. For two-particle CTQW on 1D discrete ring lattice with a large number of nodes, the time evolution of the quantum system is less affected by transmission through periodic boundary conditions and this leads to the disappearance of the cyclical behavior of the QC. In particular, the latter are still related to the interference stemming from the overlap of the wavepackets, but they turn out to be almost insensitive to the quantum statistics of the particles, a part from small fluctuations related to the discrete nature of the ring lattice.

Finally, we analyzed the building up of QC in the free- 
propagation of two identical particles in an 1D structure. Such a system can be considered the continuous space limit of an 1D ring lattice with a large number of sites and vanishing intersite distance. It provides an useful guideline both to validate the results found for the discrete case and to compare our outcomes with the analyses of nonclassical correlations appearing in scattering events in 1D semiconductor structures [28, 29]. Two different initial conditions have been examined: particles moving with the same velocity in the same or in the opposite direction. In the former case, we find that the QC of the system first show an initial increase due to the interference of the spreading single-particle wavepackets and then, for sufficiently long times, reach a stationary value, different from zero, depending upon quantum statistics. Such behavior is in qualitative agreement with the one found for CTQW in 1D ring lattice (apart from the absence of the effects related to the transmission through periodic boundary conditions and to the discreetness of the lattice). On the other hand, for the case of particles propagating one against the other, as the fermions (bosons) get closer, the degree of quantum correlation increases and attains its maximum values in correspondence of the maximum spatial overlap of the single-particle wavepackets. When the two particles get away from each other, the amount of QC decreases until it vanishes. Such a behavior appears to be consistent with the time evolution of the entanglement created in binary collisions of electrons in semiconductor structures [28, 29].

Despite the simplicity of the graphs here investigated, it seems reasonable to assume that the results obtained are representative, at least qualitatively, of the behavior of the QC between two indistinguishable particles in more complex structures, such as 2D lattices or graph with larger connectivity. Thus, the analysis here reported represents a valuable starting point to investigate the emergence of non-classical correlations in other models of or fermionic or bosonic CTWQ, where also particleparticle interaction could be considered. Furthermore, it would be of interest to insert in such models environmental noise such as the one due to lattice disorder. Indeed, the latter can be viewed as a decoherence source and, as a consequence, should result into peculiar phenomena, among which the sudden death or the revival of quantum correlations.
[1] Y. Aharonov, L. Davidovich, and N. Zagury, Phys. Rev. A 48, 1687 (1993)

[2] E. Farhi and S. Gutmann, Phys. Rev. A 58, 915 (1998)

[3] A. Ambainis, International Journal of Quantum Information 1, 507 (2003).

[4] N. Shenvi, J. Kempe, and K. BirgittaWhaley, Phys. Rev. A 67, 052307 (2003).

[5] A. Childs, E. Farhi, and S. Gutmann, Journal of Quantum Information Processing , 1 (2002).

[6] Y. Bromberg, Y. Lahini, R. Morandotti, and Y. Silberberg, Phys. Rev. Lett. 102, 253904 (2009)

[7] A. Rai, G. S. Agarwal, and J. H. H. Perk, Phys. Rev. A 78, 042304 (2008)

[8] A. Peruzzo et. al., Science 329, 1500 (2010)

[9] C. A. Ryan, M. Laforest, J. C. Boileau, and R. Laflamme, Phys. Rev. A 72, 062317 (2005).

[10] J. Du, H. Li, X. Xu, M. Shi, J. Wu, X. Zhou, and R. Han, Phys. Rev. A 67, 042316 (2003).

[11] Y. Ji, Y. Chung, D. Sprinzak, M. Heiblum, D. Mahalu, and H. Shtrikman, Nature 422, 415 (2003).

[12] I. Neder et al., Nature 448, 333 (2007).

[13] P. Samuelsson, E. V. Sukhorukov, and M. Büttiker, Phys. Rev. Lett. 92, 026805 (2004).

[14] J. Schliemann, J. I. Cirac, M. Kuś, M. Lewenstein, and D. Loss, Phys. Rev. A 64, 022303 (2001)

[15] P. Zanardi, Phys. Rev. A 65, 042101 (2002).
[16] H. M. Wiseman and J. A. Vaccaro, Phys. Rev. Lett. 91, 097902 (2003).

[17] J. R. Gittings and A. J. Fisher, Phys. Rev. A 66, 032305 (2002).

[18] M. R. Dowling, A. C. Doherty, and H. M. Wiseman, Phys. Rev. A 73, 052323 (2006)

[19] P. K. Pathak and G. S. Agarwal, Phys. Rev. A 75, 032351 (2007).

[20] S. D. Berry and J. B. Wang, Phys. Rev. A 83, 042317 (2011).

[21] S. K. Goyal and C. M. Chandrashekar, J. Phys. A 43, 235303 (2010).

[22] F. Buscemi and P. Bordone, Phys. Rev. A 84, 022303 (2011).

[23] O. Mülken and

A. Blumen, Phys. Rev. E 71, 036128 (2005).

[24] A. Volta, O. Mülken, and A. Blumen, J. Phys. A 39, 14997 (2006).

[25] X.-P. Xu, Phys. Rev. E 77, 061127 (2008)

[26] Y. Lahini, Y. Bromberg, D. N. Christodoulides, and Y. Silberberg, Phys. Rev. Lett. 105, 163905 (2010).

[27] F. Buscemi, P. Bordone, and A. Bertoni, Phys. Rev. A 75, 032301 (2007).

[28] F. Buscemi, P. Bordone, and A. Bertoni, Phys. Rev. A 73, 052312 (2006).

[29] A. Bertoni and S. Reggiani, Semiond. Sci. Technol. 19, s113 (2004). 


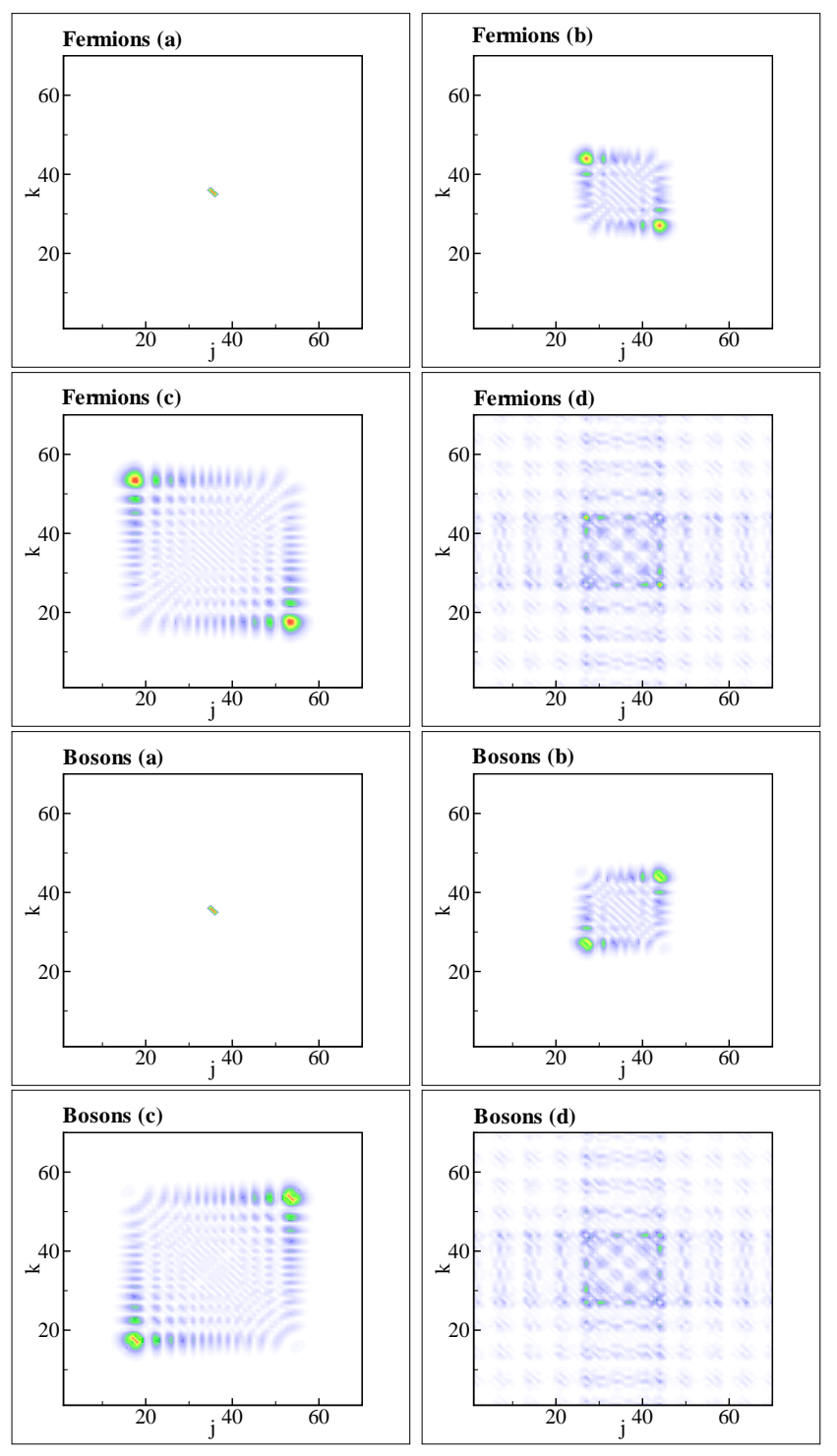

FIG. 3. Left panels: two-particle correlation function $\Gamma_{k j}^{f}$ of the two-fermion CTQW in a ring lattice with $N=70$ evaluated at four different times: $\gamma t=0,(\mathrm{a}), \gamma t=5$, (b), $\gamma t=10,(\mathrm{c})$, and $\gamma t=40(\mathrm{~d})$. The input state is $\mid \frac{N}{2} \frac{N}{2}+$ $1\rangle_{f}$. At short times, fermions exhibit ballistic evolution with spatial antibunching. At the final time, as a consequence of the interference stemming from the transmission through periodic boundary conditions, the two particle wavefunction is scattered along the whole lattice. Right panels: two-particle correlation function $\Gamma_{k j}^{b}$ of the two-boson CTQW in a ring lattice with $N=70$ evaluated at four different times: $\gamma t=0$, (a), $\gamma t=5,(\mathrm{~b}), \gamma t=10,(\mathrm{c})$, and $\gamma t=40$ (d). The input state is $\left|\frac{N}{2} \frac{N}{2}+1\right\rangle_{b}$. At short times, bosons propagate ballistically along the lattice showing spatial bunching. After reaching the boundary, the transmission through the periodic boundary conditions induces the spread of two-particle wavefunction all over the lattice. 


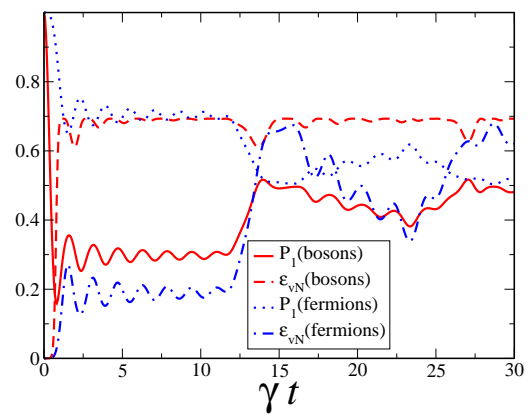

FIG. 4. Probability $P_{1}$ of finding each particle in a different subsystem and von Neumann entropy $\epsilon_{v N}$, both for two bosons and the for two fermions as a function of time for the case of a lattice ring with a number of nodes $N=50$.
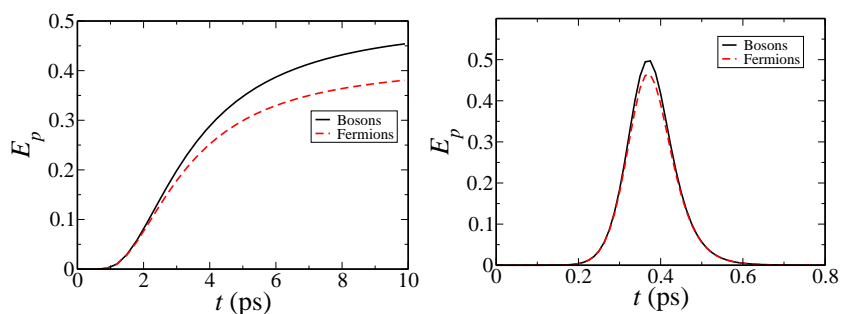

FIG. 5. (Color online) Left panel: $E_{P}$ as a function of the time for two fermions (dashed line) and two bosons (solid line) moving with the same velocity. Such a condition is equivalent to the one where the particles have zero initial kinetic energy. In order to make the numerical implementation of wavefunction dynamics simple, here we considered $E_{k}=0$. The particles are initially described by two wavepackets with initial variance $\sigma=5 \mathrm{~nm}$ and $\bar{x}_{0}=20 \mathrm{~nm}$. Right panel: $E_{P}$ as a function of the time for two fermions (dashed line) and two bosons (solid line) moving in opposite directions. The particles have the same kinetic energy $E_{k}=10 \mathrm{meV}$, and, at the initial time, are also described by two wavepackets with variance $\sigma=5 \mathrm{~nm}$ and $\bar{x}_{0}=20 \mathrm{~nm}$. In all the numerical calculations we take $m=9.1 \times 10^{-31} \mathrm{Kg}$. 